\title{
ORIENTABILITY OF MANIFOLDS FOR GENERALISED HOMOLOGY THEORIES
}

\author{
BY \\ W. C. HSIANG( ${ }^{1}$ ) AND C. T. C. WALL
}

Recently, generalised homology theories have received much attention ([5], [6], [19] etc.). An interesting question which arises in these theories is the orientability of manifolds [20]; as Whitehead remarks, this is a delicate question. In this paper we shall show that if there exists a microbundle $\xi$ over a closed manifold $M$ such that $\xi$ is $A$-orientable in the sense of Dold [6], and such that the top homology class of the Thom space $M^{\xi}$ is spherical, then $M$ is $A$-orientable in the sense of Whitehead [19]. Hence $M$ satisfies Poincaré duality with coefficient spectrum $\boldsymbol{A}$.

Let $N$ be a compact smooth manifold whose boundary $\partial N=\Sigma$ is a homotopy sphere which bounds a $\pi$-manifold: form a manifold $M$ by adjoining to $N$ a cone on its boundary $\Sigma$. We shall call such manifolds $M$ almost-smooth: the manifolds of Kervaire [11], Smale [16], Eells and Kuiper [8] (see also [18]), which do not have the same homotopy type as any closed smooth manifold, are all almost smooth. We shall show that, over any such $M$, there is a vector bundle $\xi$ such that the top homology class of its Thom complex $M^{\xi}$ is spherical. It follows that (provided $M$ is orientable in the usual sense), $M$ is orientable for $K$-theory and KO-theory [2] and for bordism theory [1]. It also follows from the existence of such bundles that the hypothesis on the signature in the theorem of Browder [4], which gives necessary and sufficient conditions for a finite, simply-connected CW-complex to have the homotopy type of a closed smooth manifold (of dimension $\neq 3,4$ ), cannot be dispensed with.

Microbundles and Thom spaces. We consider pairs $(i, j)$ of maps, with $j i$ the identity map of some fixed space $B$. Two pairs $\left(i_{1}, j_{1}\right)$ and $\left(i_{2}, j_{2}\right)$ are equivalent if there is a third pair $\left(i_{3}, j_{3}\right)$ and a commutative diagram,

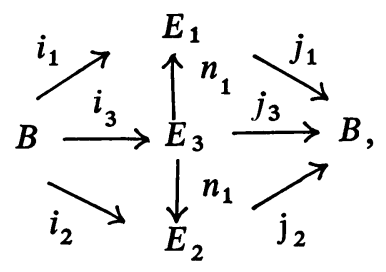

Received by the editors June 23, 1964.

(1) Partially supported by an NSF grant (GP-1853). 
where $n_{1}$ imbeds $E_{3}$ as a neighborhood of $i_{1}(B)$ in $E_{1}$ (similarly for $n_{2}$ ). A $k$-trivial pair has the form

$$
B \stackrel{i}{\rightarrow} B \times R^{k} \stackrel{j}{\rightarrow} B
$$

where $i(b)=(b, 0)$ and $j(b, x)=b$. A pair $(i, j)$ is locally $k$-trivial if $B$ has a numerable covering [7] by sets $U_{\alpha}$, with, say, $V_{\alpha}=j^{-1}\left(U_{\alpha}\right)$, such that each ( $i\left|U_{\alpha}, j\right| V_{\alpha}$ ) is equivalent to a $k$-trivial pair. A microbundle (with fibre dimension $k$ ) is an equivalence class of locally $k$-trivial pairs; we shall also use the word "microbundle" to refer to a representative pair. We call $B$ the base of the microbundle. Microbundles were invented by J. Milnor [13], [14].

Now let $(i, j)$ be locally $k$-trivial.

$$
B \stackrel{i}{\longrightarrow} E \stackrel{j}{\longrightarrow} B .
$$

We write $E_{0}=E-i(B)$, and define the Thom space $T$ of $(i, j)$ as obtained from $E$ by attaching a cone on $E_{0}$. Equivalently, $T$ is obtained from $E \times I$ by deleting $i(B) \times(0,1]$ and identifying $E_{0} \times 1$ to a point, * .

LEMMA 1. The homotopy type of $T$ depends only on the microbundle $\xi$ which is the equivalence class of $(i, j)$.

Proof. It will be enough to show that for the pairs $\left(i_{1}, j_{1}\right)$ and $\left(i_{3}, j_{3}\right)$ in (1), the map $\left(n_{1}\right)$ induced by $n_{1}$ of the corresponding $T_{3}$ in $T_{1}$ is a homotopy equivalence. Let $\lambda: E_{1} \rightarrow I$ be a map such that $\lambda i_{1}(B)=0$ and $\lambda\left(E_{1}-n_{1}\left(E_{3}\right)\right)=1$; such a map exists since $n_{1}\left(E_{3}\right)$ contains a neighborhood of $i(B)$ in $E_{1}$-we can define maps over the $U_{\alpha}$, using triviality, and piece together using the partition of unity.

Define a homotopy of the cone $C E_{1}$ by

$$
h_{t}(y, u)=(y, u+t(1-u) \lambda(y)), \quad y \in E, 0 \leqq u \leqq 1 .
$$

It is immediate that this respects the identification, also the subspace $T_{1}$ : we also use $h_{t}$ for the induced homotopy of $T_{1}$. If $y \notin n_{1}\left(E_{3}\right), \lambda(y)=1$, and $h_{1}(y, u)=(y, 1)$ $=*$. Thus the image of $h_{1}$ lies in $n_{1}\left(E_{3}\right)$ : since $n_{1}$ is an imbedding, there is a map $r: T_{1} \rightarrow T_{3}$ with $n_{1} r=h_{1}$. We assert that $r$ is a homotopy inverse to $n_{1}$; indeed, we have just defined a homotopy of $n_{1} r$ to the identity, hence there is one of $n_{1} r n_{1}$ to $n_{1}$. Since, by inspection, the image of this (i.e., of $h_{t} n_{1}$ ) is contained in the image of $n_{1}$, and $n_{1}$ is an imbedding, this gives a homotopy of $r n_{1}$ to the identity.

Q.E.D.

The lemma permits us to define (up to homotopy) the Thom space of a microbundle. Of course, this could also be done using the result of Kister [12] and Mazur that a microbundle has a canonical representative which is a bundle, but it seems more satisfactory to find a definition which does not invoke their 
constructions. If the microbundle $\xi$ has base $B$, we denote its Thom space by $B^{\xi}$.

Now suppose $\xi$ a microbundle with base $B$, let $b_{0}$ be a base point in $B$, and use $*$ as base point in $B^{\xi}$. Consider the sequence of maps

$$
E \stackrel{\Delta}{\rightarrow} E \times E \stackrel{i \times j}{\longrightarrow} B^{\xi} \times B \rightarrow B^{\xi} \# B,
$$

where $\Delta$ is the diagonal map, $i$ the inclusion, and the last map collapses the axes. The composite is null-homotopic on $E_{0}$ : we define a null-homotopy by

$$
f_{t}(y)=((y, t), j(y)),
$$

and this extends the composite to a map $\phi: B^{\xi} \rightarrow B^{\xi} \# B$. It is clear that the homotopy class of $\phi$ depends only on $\xi$, and that $\phi$ is defined everywhere by $\phi(y, t)=((y, t), j(y))$.

Attaching a cell. We shall now suppose that $B$ is formed by attaching an $m$-cell to a space $K: B=K \cup e_{m}$. Let $c: B \rightarrow S^{m}$ be the collapsing map, defined by shrinking $K$ to a point. Let 0 be an interior point of $e_{m}$. Now let $\xi$ be a microbundle,

$$
B \stackrel{i}{\longrightarrow} E \stackrel{j}{\longrightarrow} B .
$$

Then $i(0)$ has a neighborhood in $E$ homeomorphic to an open set in euclidean space $\boldsymbol{R}^{k} \times \boldsymbol{R}^{m}$. Indeed, if we consider the induced microbundle over $D^{m}$, it must be trivial, so $i\left(D^{m}\right)$ has a neighborhood $D^{k} \times D^{m}$ there. But we can suppose $D^{m}$ imbedded (e.g., as a cell of half the radius), and the induced microbundle is just the restriction. Now shrink all outside $D^{k} \times D^{m}$ to a point; this defines a map $\gamma: E \rightarrow S^{k+m}$, and $\gamma\left(E_{0}\right)$ does not contain $\gamma i(0)$. Thus there is an extension of $\gamma$ over $C E_{0}$, unique up to homotopy, in the contractible space $S^{k+m}-\gamma i(0)$. We write $\Gamma: B^{\xi} \rightarrow S^{k+m}$ for the map so defined.

REMARK. It is easy to see that $B^{\xi}$ is (up to homotopy) obtained from $K^{\xi}$ by attaching a $(k+m)$-cell, and that $\Gamma$ corresponds to shrinking $K^{\xi}$ to a point. Thus if $B$ is a finite $C W$-complex, so (up to homotopy) is $B^{\xi}$, with cell dimensions increased by $k$.

The restriction of $\xi$ to 0 is trivial, so its Thom space has the homotopy type of a sphere $S^{k}$. We write $f: S^{k} \rightarrow B^{\xi}$ for the map induced by the inclusion of 0 in $B$. (Orientations in $D^{k}, D^{m}$, and the constructed spaces $D^{k} \times D^{m}, S^{k+m}, S^{k}$ are supposed consistent throughout.)

LEMMA 2. The following diagram is homotopy commutative:

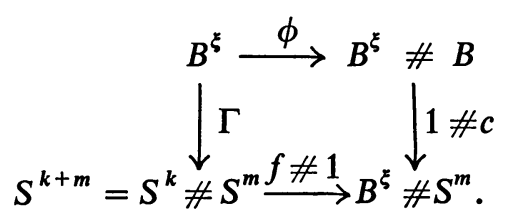


Proof. Recall that $\phi$ is defined by $\phi(y, t)=((y, t), j(y))$, so $(1 \# c) \phi(y, t)$ $=((y, t), c j(y))$ is at the base point if $j(y) \in K$. So $(1 \# c) \phi$ can be factored through the space obtained from $B^{\xi}$ by shrinking $K^{\xi}$ to a point, i.e., through $\Gamma$. Now on the $D^{k} \times D^{m}$ used in defining $\Gamma$ our map becomes

$$
(1 \# c) \phi((p, q), 0)=\{((p, q), 0), c(q)\} \text {. }
$$

So, in the factorised map of $S^{k} \# S^{m}$ to $B^{\xi} \# S^{m}$, the second component is mapped by the identity, and we have the $m$-fold suspension of the induced map on the first component-which is just the inclusion of the Thom space over 0 , i.e., the $\operatorname{map} f$.

Q.E.D.

Homology theories. A spectrum $\boldsymbol{E}$ is a sequence $\left\{E_{n}: n \in Z\right\}$ of spaces together with a sequence of maps $\varepsilon_{n}: S E_{n} \rightarrow E_{n+1}$. Following Whitehead [19], we define generalised homology and cohomology groups with coefficients in $E$ as follows:

$$
\begin{aligned}
& h_{n}(X, A ; E)=\pi_{n}(X / A \not E)=\lim _{i} \pi_{n+i}\left(X / A \# E_{i}\right), \\
& h^{n}(X, A ; E)=\pi_{n}(\boldsymbol{F}(X / A, E))=\lim _{i} \pi_{-n+i}\left(F\left(X / A, E_{i}\right)\right),
\end{aligned}
$$

where $F\left(X / A, E_{i}\right)$ denotes the function space of maps of $X / A$ into $E_{i}$, so $F$ is a covariant functor of $E_{i} ; F$ is the induced functor on spectra; and $\lim _{i}$ means the direct limit of groups. It follows from the definition that $h_{*}(; E)$ and $h^{*}(; E)$ are functors defined on the category of $s$-homotopy classes of $s$-maps to the category of graded abelian groups. These functors satisfy all the EilenbergSteenrod axioms for homology theories except the dimension axiom [9], [10], [19].

Now let $S=\left\{S^{n}, \sigma_{n}\right\}$ be the sphere spectrum, where $\sigma_{n}: S S^{n} \rightarrow S^{n+1}$ is the identity map. Let $\boldsymbol{A}$ be another spectrum and $\mathrm{\imath}: \boldsymbol{S} \rightarrow \boldsymbol{A}$ a map of spectra (that is, a sequence of maps $i_{n}: S^{n} \rightarrow A_{n}$ with $\left.i_{n+1} \sigma_{n}=\alpha_{n}\left(S_{t_{n}}\right)\right)$. Then, following Dold [6], we call a microbundle $\xi$ with base $B$ and fibre dimension $k$ A-orientable if there is a class $U \in h^{k}\left(B^{\xi} ; A\right)$ such that $f^{*} U \in h^{k}\left(S^{k} ; A\right)$ is the class determined by $\mathfrak{\imath}_{k}: S^{k} \rightarrow A_{k}$, or, equivalently, is the image under $\imath$ of the $k$-fold suspension of the unit class in $h^{0}\left(S^{0} ; S\right)$.

We call $U$ a fundamental class for the microbundle. If we also write $U$ for a representative $s$-map $B^{\xi} \rightarrow S^{k} A$, the condition becomes $U f \simeq{ }_{s} S^{k}$.

Now suppose (as above) that $B=K \cup e_{m}$. Then we call $B$ A-orientable if there is a class $z \in h_{m}(B ; A)$ whose Kronecker product with the class defined by $c$ in $h^{m}(B ; S)$ is $\mathbf{~}$. In the case when $B$ is a triangulated homology manifold, and $e_{m}$ is a simplex of top dimension, this coincides with the definition given by Whitehead [19].

Again we call $z$ a fundamental class, write $z$ for a representative $s$-map $S^{m} S \rightarrow B \# A$, and note that the condition may be rewritten as $(c \# 1) z \simeq{ }_{s} S^{m}$. 
THEOREM 3. Suppose $B=K \cup e_{m}$, $\xi$ is a microbundle with base $B$, and (with the notations above) (i) $\Gamma$ has an (s-homotopy) right inverse $\alpha$, (ii) $\xi$ is A-orientable. Then $B$ is A-orientable.

Proof. Define $S^{k} z$ as the composite of

$$
S^{k+m} \stackrel{\alpha}{\rightarrow} B^{\xi} \stackrel{\phi}{\rightarrow} B^{\xi} \# B \stackrel{U \# 1}{\longrightarrow} S^{k} A \not \# B \rightarrow S^{k} B \# A .
$$

Then the Kronecker product of $z$ with $c$ is represented by

$$
\begin{aligned}
(1 \# c)(U \# 1) \phi \alpha & =(U \# 1)(1 \# c) \phi \alpha & & \\
& \simeq(U \# 1)(f \# 1) \Gamma \alpha & & \text { by Lemma } 2, \\
& \simeq{ }_{s} U f \# 1 & & \text { by (i), } \\
& \simeq{ }_{s} S^{k}{ }_{\imath} \# 1 & & \text { by (ii), }
\end{aligned}
$$

and the result follows by putting the factors in the right order. If the reader is ill at ease about signs, he may observe that we could (if necessary) change the sign in the definition of $z$ to put things right.

Q.E.D.

For any spectrum $\boldsymbol{A}$, there are natural pairings $\lambda: S \# \boldsymbol{A} \rightarrow \boldsymbol{A}$ and $\lambda^{\prime}: \boldsymbol{A} \# \boldsymbol{S} \rightarrow \boldsymbol{A}$ defined by the composite maps

$$
\begin{aligned}
& S^{p} \# A_{q} \stackrel{S^{p-1} \alpha_{q}}{\longrightarrow} S^{p-1} \# A_{q+1} \longrightarrow \cdots \stackrel{\alpha_{p+q-1}}{\longrightarrow} A_{p+q} \quad\left(\lambda_{p, q}\right), \\
& A_{q} \# S^{p} \stackrel{\tau}{\longrightarrow} S^{p} \# A_{q} \stackrel{\lambda_{p, q}}{\longrightarrow} A_{p+q}
\end{aligned}
$$

where $\tau$ interchanges the factors. The spectrum $\boldsymbol{A}$ is called a ring spectrum if there is a pairing $\rho: A \not A \rightarrow A$ and a map $\mathrm{\imath}: S \rightarrow \boldsymbol{A}$ such that the diagram

is homotopy commutative.

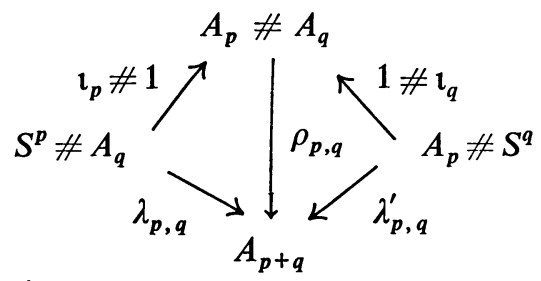

Let $M$ be a (compact, unbounded) topological manifold or triangulated homology manifold. We can always write $M=N \cup D^{m}$ by imbedding a disc or simplex and (for $N$ ) deleting its interior. The shrinking map $c: M \rightarrow S^{m}$ determines the top cohomology class of $M$. If $\xi$ satisfies the two conditions of Theorem 1 , then $M$ is $A$-orientable. Using Theorem 7.4 of [19], we now have

THEOREM 4. Let $M$ be a (compact, unbounded) triangulated homology manifold, $\boldsymbol{A}$ a ring spectrum, and $\xi$ an A-orientable microbundle with base $M$. If the top homology class of $M^{\xi}$ is spherical, then $M$ satisfies Poincaré duality with coefficient spectrum $\boldsymbol{A}$. 
Observe that our hypothesis requires, in particular, that a top homology class exist, i.e. (if $M$ is nonorientable), that on traversing a loop in $M$, the orientation of the fibre of $\xi$ changes if and only if that of $M$ does.

Now let $G$ be a subgroup of the infinite orthogonal group $O$. For $G_{n}=G \cap O_{n}$, there is an associated vector bundle $v_{n}$ over the classifying space $B G_{n}$. Consider the sequence of the Thom complexes $\left\{M G_{n}=\left(B G_{n}\right)^{v_{n}}: n \in Z^{+}\right\}$. The classifying map of the Whitney sum of $v_{n}$ and a trivial line bundle induces a map $\varepsilon_{n}: S M G_{n} \rightarrow M G_{n+1}$. The sequence $\left\{M G_{n}, \varepsilon_{n}\right\}$ is the classical Thom spectrum $M G$ [17]. Following Atiyah [1], the homology theory with coefficients in $M G$ is called the bordism theory with the structure group $G$. In the most familiar cases (e.g., $G=O, S O, U, S U, S p, 1), G_{m} \times G_{n} \subset G_{m+n}$, so the Whitney sum of $v_{m}$ and $v_{n}$ is a $G_{m+n}$-bundle, and we obtain maps $M G_{m} \# M G_{n} \rightarrow M G_{m+n}$. The inclusion $1 \subset G$ also induces a map $S=M 1 \rightarrow M G$; with respect to these maps, $M G$ is a ring spectrum.

LEMMA 5. If $\xi$ is a vector bundle with structure group $G_{k}$, it is MG-orientable.

Proof. The classifying map $f: \xi \rightarrow v_{k}$ induces a map of Thom complexes $\hat{f}: B^{\xi} \rightarrow M G_{k}$. This represents a cohomology class $U \in h^{k}\left(B^{\xi} ; M G\right)$. The restriction to a fibre gives the composition $S^{k} \subset B^{\xi} \rightarrow M G_{k}$, i.e., the map $M 1_{k} \rightarrow M G_{k}$ induced by the inclusion $1_{k} \subset G_{k}$. So $U$ is indeed a fundamental class. Q.E.D.

Orientability of almost-smooth manifolds. If $M^{m}$ is a closed, smooth manifold, there is a smooth imbedding $f: M^{m} \rightarrow S^{2 m+1}$. Let $v$ be the normal bundle of the imbedding. Then by shrinking the complement of a tubular neighbourhood of $f(M)$ to a point, we obtain a map $\alpha: S^{2 m+1} \rightarrow M^{v}$ (this is the Pontrjagin-Thom construction [15], [17]). With $c, \Gamma$ as above, it is clear that $\Gamma_{\alpha}$ has degree 1 , so is homotopic to the identity. Theorem 3 now shows that if $v$ is orientable for any homology theory, then so is $M$. This simplifies the problem, as it is easier to study $\boldsymbol{A}$-orientability of vector bundles than of manifolds. We now show that a similar reduction can sometimes be effected even if $M$ is not smooth.

Recall that we call $M$ almost smooth if $M=N \cup C \Sigma$ where $N$ is smooth and $\partial N=\Sigma$ is a homotopy sphere which bounds a $\pi$-manifold.

THEOREM 6. Let $M$ be a closed, almost-smooth manifold. Then there is a vector bundle $\xi$ over $M$ such that the top homology class of $M^{\xi}$ is spherical.

We give two proofs of this result.

First proof. Let $\Sigma=\partial H$, where $H$ is a $\pi$-manifold. Define $W$ by gluing $H$ to $N$ along $\Sigma$. Then $W$ is smooth and $W / H \cong N / \Sigma$ is homeomorphic to $M$. Let $\eta$ be the stable normal bundle of $W$. Then $\eta \mid H$ is trivial, since $H$ is a $\pi$-manifold. Hence $\eta$ is induced from some bundle $\xi$ on $W / H \cong M$. Now $W^{\eta}$ maps to $M^{\xi}$ by the collapsing map, which has degree 1 ; since the top homology class of $W^{\eta}$ is spherical, so is that of $M^{\xi}$. 
Second proof. First forget that $\Sigma$ bounds a $\pi$-manifold. Now $N$ is smooth; if $\eta^{k}$ is a stable normal bundle, there is an element of $\pi_{m+k}\left(N^{\eta}, \Sigma^{\eta}\right)$ of degree 1 for we may imbed $(N, \Sigma)$ in $\left(D^{m+k}, \partial D^{m+k}\right)$, and again use the Pontrjagin-Thom construction. Now $\eta \mid \Sigma$ is trivial: choose a framing, then $\eta$ is induced by a map of $\Sigma$ to a point. So the map $N \rightarrow N / \Sigma \cong M$ is covered by a bundle map of $\eta$ to, say, $\xi$. The above homotopy element maps to $\pi_{m+k}\left(M^{\xi}, S^{k}\right)$. Hence, by the homotopy exact sequence, it lifts to an element of $\pi_{m+k}\left(M^{\xi}\right)$ if and only if the image in $\pi_{m+k-1}\left(S^{k}\right)$ vanishes. But according to Pontrjagin [15], the existence of a framing of $v \mid \Sigma$ such that the corresponding element of $\pi_{m+k-1}\left(S^{k}\right)$ vanishes is equivalent to $\Sigma$ bounding a $\pi$-manifold.

Q.E.D.

CoRollary. Suppose $M$ closed and almost smooth. If $M$ is orientable, it satisfies Poincaré duality in bordism theory (with coefficient spectrum MSO); if also $w_{2}(M)=0, M$ satisfies Poincaré duality for K-theory and KO-theory.

Proof. Let $\xi$ be the vector bundle given by Theorem 6 . If $M$ is orientable, so is $\xi$, and by Lemma $5, \xi$ is $\boldsymbol{M S O}$-orientable. If also $w_{2}(M)=0$, then we say $w_{2}(\xi)=0$, so the group of $\xi$ can be lifted to the spinor group. Hence $\xi$ is $K$ and KO-orientable [3]. The corollary now follows immediately from Theorem 4.

It remains to check the relation between Stiefel classes of $M$ and those of $\xi$. We work with (ordinary) mod 2 cohomology, and the Thom isomorphism $\Phi$, the composite of

$$
H^{r}(M) \stackrel{\otimes U}{\longrightarrow} H^{r+k}\left(M^{\xi} \# M\right) \stackrel{\phi^{*}}{\longrightarrow} H^{r+k}\left(M^{\xi}\right) .
$$

Let us suppose $M$ (hence $\xi$ ) orientable, so $S q^{1} U=0$. Then $S q^{2} U=U w_{2}(\xi)$, by definition; likewise, for $u \in H^{m-2}(M), S q^{2} u=u w_{2}(M)$. Hence

$$
\begin{aligned}
S q^{2} \Phi(u) & =\phi^{*} S q^{2}(u \otimes U) \\
& =\phi^{*}\left\{u w_{2}(M) \otimes U+0+u \otimes U w_{2}(\xi)\right\} \\
& =u U\left(w_{2}(M)+w_{2}(\xi)\right) \\
& =\Phi\left\{u\left(w_{2}(M)+w_{2}(\xi)\right)\right\} .
\end{aligned}
$$

But this vanishes, since the top class of $M^{\xi}$ is spherical. So for all $u \in H^{m-2}(M), u\left(w_{2}(M)+w_{2}(\xi)\right)=0$. Hence, by $(\bmod 2)$ duality, $w_{2}(M)=w_{2}(\xi)$. We could similarly check all the usual relations between Stiefel classes of $M$ and those of $\xi$.

Q.E.D.

We now observe that, as noted in the introduction, several almost-smooth manifolds are known which do not have the homotopy type of any smooth manifold. But by Theorem 6, over any one exists a bundle, the top class of whose Thom space is spherical. So the hypothesis of Browder [4] concerning the signature is essential. Indeed, combining Browder's arguments with our Theorem 6, we obtain at once 
THEOREM 7. Suppose $X$ a simply connected, finite CW-complex, which satisfies Poincaré duality with a fundamental class $g \in H_{n}(X), n \neq 4$. Then $X$ has the homotopy type of an almost-smooth manifold if and only if there is a vector bundle $\xi$ over $X$, with $\Phi(g)$ spherical.

\section{REFERENCES}

1. M. F. Atiyah, Bordism and cobordism, Proc. Cambridge Philos. Soc. 57 (1961), 200-208.

2. M. F. Atiyah and F. Hirzebruch, Vector bundles and homogeneous spaces, Proc. Sympos. Pure Math. Vol. 3, pp. 7-38, Amer. Math. Soc., Providence, R. I., 1961.

3. M. F. Atiyah, R. Bott and A. Shapiro, Clifford modules, Topology 3 (suppl. 1) (1964), 3-38.

4. W. Browder, Homotopy type of differentiable manifolds, Notes, Aarhus Colloquium on Algebraic Topology, Aarhus, 1962.

5. E. H. Brown, Cohomology theories, Ann. of Math. (2) 75 (1962), 467-484.

6. A. Dold, Relations between ordinary and extraordinary cohomology, Notes, Aarhus Colloquium on Algebraic Topology, Aarhus, 1962.

7. - Partitions of unity in the theory of fibrations, Ann. of Math. (2) 78 (1963), 223-255.

8. J. Eells and N. H. Kuiper, Manifolds which are like projective planes, Inst. Hautes Études Sci. Publ. Math. No. 14, 1962.

9. S. Eilenberg and N. Steenrod, Axiomatic approach to homology theory, Proc. Nat. Acad. Sci. U.S.A. 31 (1945), 117-12.0.

10. —_ Foundations of algebraic topology, Princeton Univ. Press, Princeton, N. J., 1952.

11. M. Kervaire, A manifold which does not admit any differential structure, Comment. Math. Helv. 34 (1960), 257-270.

12. J. M. Kister, Microbundles are fibre bundles, Bull. Amer. Math. Soc. 69 (1963), 854-857.

13. J. Milnor, Microbundles, Mimeographed, Princeton Univ., Princeton, N. J., 1961.

14. - Topological manifolds and smooth manifolds, Proc. Internat. Congr. Math., pp. 132-138, Stockholm, 1962.

15. L. Pontrjagin, Smooth manifolds and their applications in homotopy theory, Trudy Mat. Inst. Steklov. 45 (1955), 139 pp.; Amer. Math. Soc. Transl. (2) 11 (1959), 1-114.

16. S. Smale, Generalized Poincaré's conjecture in dimensions greater than four, Ann. of Math. (2) 74 (1961), 391-406.

17. R. Thom, Quelques propriétés globales des variétés differentiables, Comment. Math. Helv. 28 (1954), 17-86.

18. C. T. C. Wall, Classification of (n-1)-connected $2 n$-manifolds, Ann. of Math. (2) 75 (1962), 163-189.

19. G. W. Whitehead, Generalized homology theories, Trans. Amer. Math. Soc. 102 (1962), 227-283.

20. - Some aspects of stable homotopy theory, Notes, Aarhus Colloquium on algebraic Topology, Aarhus, 1962.

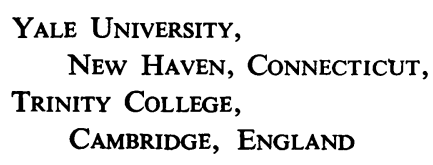

